Gynaecologia 1961;151:111-112

\title{
Das Rokitansky-Küster-Syndrom
}

Uterus bipartitus solidus rudimentarius cum vagina solida

\begin{tabular}{|l|l|}
\hline G.A. & Hauser \\
\hline M. & Keller \\
\hline Th. & Koller \\
\hline R. & Wenner \\
\hline
\end{tabular}

Universitäts-Frauenklinik Basel (Direktor: Prof. Th. Koller)

Vom bereits an der Herbsttagung der Schweizerischen Gesellschaft für Gynäkologie vom 23. 11. 1957 in Zurich von uns beschriebenen und benannten Rokitansky-Küster-Syndrom werden 11 operativ veri-fizierte Fälle in Bildern demonstriert.

Das Syndrom besteht aus folgenden Symptomen:

Vaginalaplasie mit normaler Vulva, jedoch weit hinten stehender, großer und oft klaffender Urethra (cave Verwechslung mit Introitus!).

Äußerlich unauffällig weiblicher Habitus mit gut ausgebildeten sekundären

Geschlechtsmerkmalen (Mammae und Sexualbehaarung).

Uterusdoppelmißbildung (in der Regel ohne Lichtung), kranial kolbenförmig und nach medial kommaförmig auslaufend (Abb. 1).

Schlanke Tuben und normal große, hochstehende Ovarien (feh-lender Descensus ovarii) (Abb. 1)

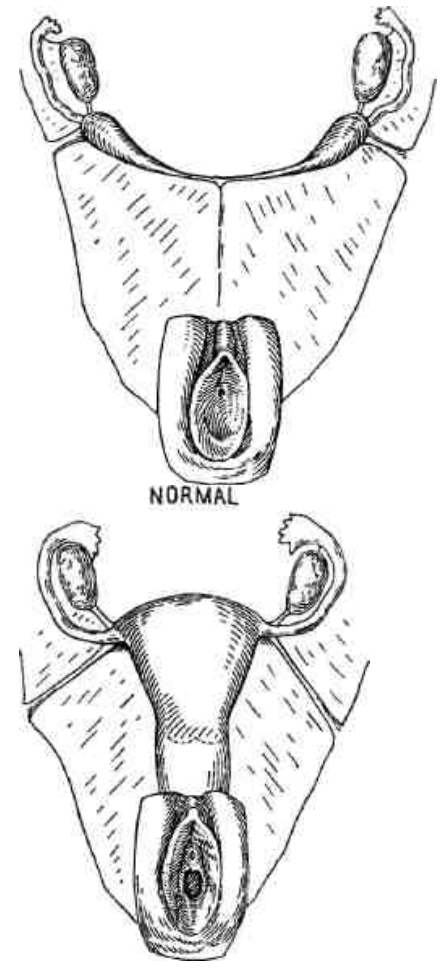


Abb. 1. Schema des Rokitansky-Küster-Syndroms (rechts): Aplasie der Vagina, Uterus gedoppelt und nach lateral kolbig aufgetrieben, Tuben und Ovarien normal.

112

Hauser, Keller, Koller, Wenner

Normale Hormonausscheidung und chromosomal «weibliches» Geschlecht.

Oft Kombination mit anderen Mißbildungen (2 Fälle mit ein-seitiger Nierenaplasie, 1 Fall mit Aortenaneurysma).

Das Auffallendste an diesem Syndrom ist das Zunebmen deГ Hem-mungsmißbildung von kranial nach caudal (in kranialem Anted Uterus gedoppelt, caudal nur faserförmig, Fehlen der Vagina). Üblicher-weise nehmen die Doppelmißbildungen nach kaudal hin an Stärke ab.

Bei den meisten Fallen mit Vaginalaplasie findet sich dieser typische Situs, ausgenommen die Fälle mit «testikulärer Femininisierung», die aber chromosomal und gonodal männlich sind. Eine ausführliche Publikation (gemeinsam mit Schreíner, Zurich) ist in Vorbereitung.

Líteraturverzeichnis

Hauser, G. A.; Keller, M.; Koller, T. und Wenner, R.: Eine typische Stufe der Genitalmißbildung. Gynaecologia 145: 332 (1958). Küster, H.: Uterus bipartitus solidvis rudimentarius cum vagina solida. Z. Geburtsli.

Gynäk. 67: 692 (1910). Rokítansky: Uber sog. Verdoppelung des Uterus. Med. Jahrb. des Osterreich.

Staates 26: 39 (1838). 